\title{
Hiding Information in Noise: Fundamental Limits of Covert Wireless Communication
}

\author{
Boulat A. Bash, Student Member, IEEE, Dennis Goeckel, Fellow, IEEE, Saikat Guha, Member, IEEE \\ and Don Towsley, Fellow, IEEE
}

\begin{abstract}
Widely-deployed encryption-based security prevents unauthorized decoding, but does not ensure undetectability of communication. However, covert, or low probability of detection/intercept (LPD/LPI) communication is crucial in many scenarios ranging from covert military operations and the organization of social unrest, to privacy protection for users of wireless networks. In addition, encrypted data or even just the transmission of a signal can arouse suspicion, and even the most theoretically robust encryption can often be defeated by a determined adversary using non-computational methods such as sidechannel analysis. Various covert communication techniques were developed to address these concerns, including steganography for finite-alphabet noiseless applications and spread-spectrum systems for wireless communications. After reviewing these covert communication systems, this article discusses new results on the fundamental limits of their capabilities, as well as provides a vision for the future of such systems.
\end{abstract}

\section{INTRODUCTION}

Security and privacy are critical in modern-day wireless communication. Widely-deployed conventional cryptography presents the adversary with a problem that he/she is assumed not to be able to solve because of computational constraints, while information-theoretic secrecy presents the adversary with a signal from which he/she cannot extract information about the message contained therein. However, while these approaches address security in many domains by protecting the content of the message, they do not mitigate the threat to users' privacy from the discovery of the very existence of the message itself.

Indeed, transmission attempts expose connections between the parties involved, and recent disclosures of massive surveillance programs revealed that this "metadata" is widely collected. Furthermore, the transmission of encrypted data can arouse suspicion, and many cryptographic schemes can be defeated by a determined adversary using non-computational means such as side-channel analysis. Anonymous communication tools such as Tor resist metadata collection and traffic analysis by randomly directing encrypted messages through a large network. While these tools conceal the identities of source and destination nodes in a "crowd" of relays, they are designed for the Internet and are not effective in wireless networks, which are typically orders of magnitude smaller.

Boulat A. Bash (corresponding author) and Saikat Guha are with Raytheon BBN Technologies.

Dennis Goeckel and Don Towsley are with the University of Massachusetts, Amherst.

This research was sponsored by the National Science Foundation under grants CNS-1018464 and ECCS-1309573.

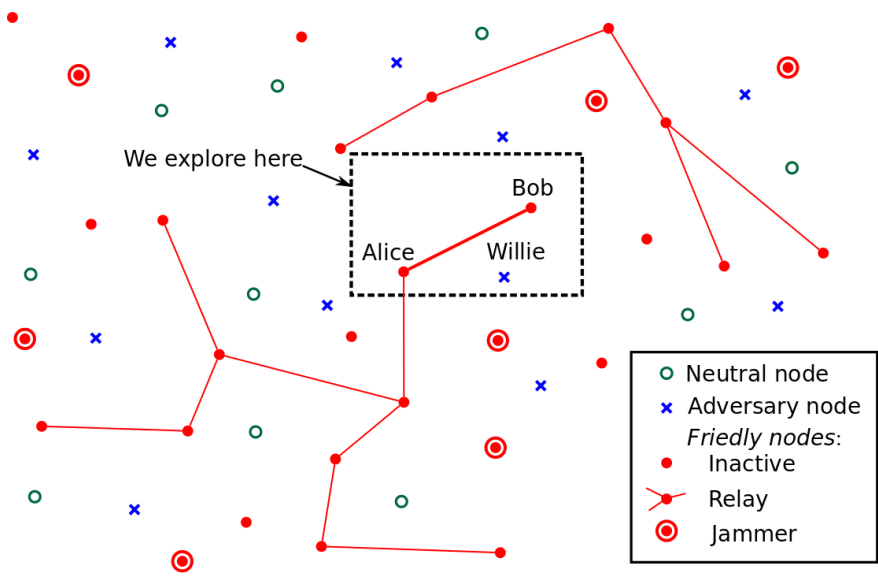

Fig. 1: Our vision of a "shadow network". Most of this article focuses on the scenario involving the indicated three nodes: transmitter Alice, receiver Bob, and warden Willie.

Moreover, such tools offer little protection to users whose communications are already being monitored by the adversaries. Thus, secure communication systems should also provide covert, stealth, or low probability of detection/intercept (LPD/LPI) communication. Such systems not only protect the information contained in the message from being decoded, but also prevent the adversary from detecting the transmission attempt in the first place and allow communication where it is prohibited.

The overarching goal of covert wireless communication research is the establishment of "shadow networks" like that depicted in Figure 1. They are assembled from relays that generate, transmit, receive and consume data, and jammers that generate artificial noise and impair the ability of wardens to detect the presence of communication (we discuss the details of this vision in Section IV]. However, to create such networks, we must first learn how to connect their component nodes by stealthy communication links. Therefore, in this article we focus on the fundamental limits of such point-to-point links and address the following question: how much information can a sender Alice reliably transmit (if she chooses to transmit) to the intended recipient Bob while hiding it from the adversary, warden Willie?

We begin in Section II by briefly reviewing the field of steganography, or the practice of hiding messages in innocuous objects. Steganography is important as it was arguably the first covert communication method devised by man. More recently it has been extensively studied by both the computer science 
and information theory communities in the context of hiding information in digital media. However, since steganography enables covert communication only at the application layer, its analysis has limited use for physical layer covert communication techniques such as spread-spectrum. Therefore, in Section III we examine the fundamental limits of covert communication over analog radio-frequency (RF) channels, where the information is hidden in the channel artifacts such as additive white Gaussian noise (AWGN), as well as digital communication channels, and briefly touch upon the covert broadcast scenario at the end of the section. We conclude in Section IV] with a discussion of shadow networks and ongoing research in jammer-assisted covert communication.

\section{STEGANOGRAPHY}

Covert communication is an ancient discipline: a description of it is given by Herodotus circa $440 \mathrm{BCE}$ in The Histories, an account of the Greco-Persian Wars: in Chapter 5 Paragraph 35 , Histiaeus shaves the head of his slave, tattoos the message on his scalp, waits until the hair grows back, and then sends the slave to Aristagoras with instructions to shave the head and read the message that calls for an anti-Persian revolt in Ionia; in Chapter 7 Paragraph 239, Demaratus warns Sparta of an imminent Persian invasion by scraping the wax off a wax tablet, scribbling a message on the exposed wood, and concealing the message by covering the tablet with wax. This practice of hiding sensitive messages in innocuous objects is known as steganography.

Modern digital steganography conceals messages in finitelength, finite-alphabet covertext objects, such as images or software binary code. Embedding hidden messages in covertext produces stegotext, necessarily changing the properties of the covertext. The countermeasure for steganography, steganalysis (an analog of cryptanalysis for cryptography), looks for these changes. Covertext is usually unavailable for steganalysis (when it is, steganalysis consists of the trivial comparison between the covertext and the suspected stegotext). However, Willie is assumed to have a complete statistical model of the covertext. The amount of information that can be embedded without being discovered depends on whether Alice also has access to this model. If she does, then positive-rate steganography is achievable: given an $\mathcal{O}(n)$-bit 1 secret "key" that is shared with Bob prior to the embedding, $\mathcal{O}(n)$ bits can be embedded in an $n$-symbol covertext without being detected by Willie [1, Chapter 13.1].

Recent work focuses on the more general scenario where the complete statistical model of the covertext is unavailable to Alice. Then, Alice can safely embed $\mathcal{O}(\sqrt{n} \log n)$ bits by modifying $\mathcal{O}(\sqrt{n})$ symbols out of $n$ in the covertext, at the cost of pre-sharing $\mathcal{O}(\sqrt{n} \log n)$ secret bits with Bob. Note that this square root law of digital steganography yields zero-rate steganography since $\lim _{n \rightarrow \infty} \frac{\mathcal{O}(\sqrt{n} \log n)}{n}=0$ bits/symbol. The proof is available in Chapter 13.2.1 of the review of pre-2009 work in digital steganography [1]. More recent work shows that an empirical model of covertext

\footnotetext{
${ }^{1}$ We use the Big-O notation in this article, where $\mathcal{O}(f(n))$ denotes an asymptotic upper bound.
}

suffices to break the square root law and achieve positiverate steganography [2]. Essentially, while embedding at a positive rate lets Willie obtain $\mathcal{O}(n)$ stegotext observations (enabling detection of Alice when statistics of covertext and stegotext differ), the increasing size $n$ of the covertext allows Alice to improve her covertext model and produce statisticallymatching stegotext.

However, steganography is inherently an application layer covert communication technique. As such, the results for steganography have limited use in physical layer covert communication. First, analysis of the steganographic systems generally assumes that stegotext is not corrupted by a noisy channel. Second, the generalization of the results for steganographic systems is limited because of their finite-alphabet discrete nature. Third, by embedding the hidden messages, Alice replaces part of the covertext. While this effectively enables the recent positive rate steganography methods [2], it cannot be done in standard communication systems unless Alice controls Willie's noise source. Finally, the most serious drawback of using steganography for covert communication is the necessity of transmitting the stegotext from Alice to Bob-a potentially unrealizable requirement when all communication is prohibited. We thus consider physical layer covert communication that employs channel artifacts such as noise to hide transmissions.

\section{Physical LAYER COVERT COMMUNiCATION}

We begin the investigation of physical layer covert communication by considering RF wireless communication. Since its emergence in the early 20th century, protecting wireless RF communication from detection, jamming and eavesdropping has been of paramount concern. Spread spectrum techniques, devised between the two world wars to address this issue, have constituted the earliest and, arguably, the most enduring form of physical layer security.

\section{A. Spread Spectrum Communication}

Essentially, the spread spectrum approach involves transmitting a signal that requires a bandwidth $W_{M}$ on a much wider bandwidth $W_{s} \gg W_{M}$, thereby suppressing the power spectral density of the transmission below the noise floor. Spread spectrum systems provide both a covert communication capability as well as resistance to jamming, fading, and other forms of interference. A comprehensive review of this field is available in [3]. Typical spread spectrum techniques include direct sequence spread spectrum (DSSS), frequency-hopping spread spectrum (FHSS), and their combination.

When Alice uses DSSS, she multiplies the signal waveform by the spreading sequence-a randomly-generated binary waveform with a substantially higher bandwidth than the original signal. The resulting waveform is thus "spread" over a wider bandwidth, which reduces the power spectral density of the transmitted signal. Bob uses the same spreading sequence to de-spread the received waveform and obtain the original signal. The spreading sequence is exchanged by Alice and 


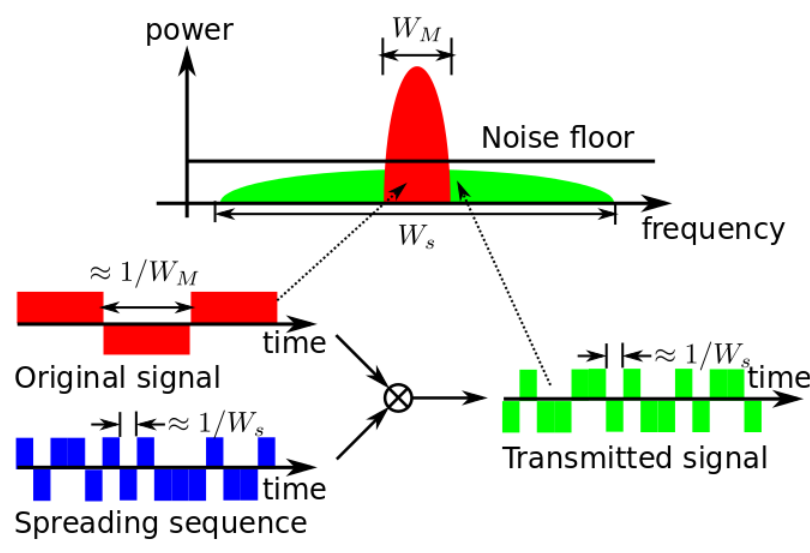

(a) DSSS

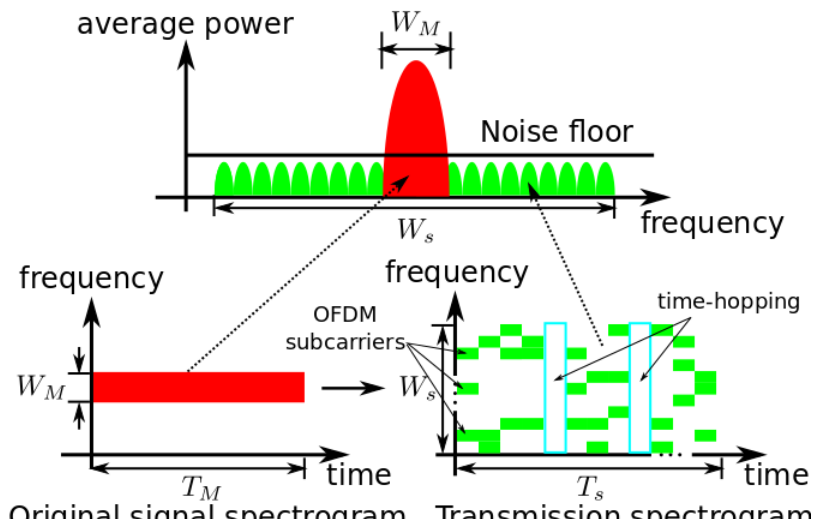

Original signal spectrogram Transmission spectrogram

(b) FHSS with OFDM and time-hopping.

Fig. 2: Spread spectrum techniques.

Bob prior to transmission and is kept secret from Willie 2 Outside of security applications, the use of public uncorrelated spreading sequences between transmitter/receiver pairs enables multiple access; DSSS thus forms the basis of code-division multiple access (CDMA) protocols used in cellular telephony. The operation of DSSS is illustrated in Figure 2 a a .

When Alice uses FHSS, she re-tunes the carrier frequency for each transmitted symbol. However, like the spreading sequence in DSSS, the frequency-hopping pattern is also randomly generated and secretly shared between her and Bob prior to the transmission. FHSS can be combined with orthogonal frequency-division multiplexing (OFDM), enabling the use of multiple carrier frequencies. To further reduce the average transmitted symbol power, FHSS can be used with time-hopping techniques that randomly vary the duty cycle (the time-hopping pattern is also secretly pre-shared between Alice and Bob prior to the transmission). The operation of FHSS with OFDM and time-hopping is illustrated in Figure 2.b.

Although spread spectrum architectures are well-developed, the analytical evaluation of covert communication has been sparse. A. Hero studied secrecy as well as undetectability [4] in a multiple-input multiple-output (MIMO) setting, focusing on the signal processing aspects. He recognized that covert communication systems are constrained by average power, and noted the need to explore the fundamental informationtheoretic limits in the conclusion of his work. In fact, knowledge of the limits of any communication system is important, particularly since modern coding techniques (such as Turbo codes and low-density parity check codes) allow $3 \mathrm{G} / 4 \mathrm{G}$ cellular systems to operate near their theoretical channel capacity, the maximum rate of reliable communication that is unconstrained by the security requirements. However, while the secrecy portion of [4] has drawn significant attention, the covert communication portion has been largely overlooked until our work on the square root limit of covert communication

\footnotetext{
${ }^{2}$ While an exchange of a secret prior to covert communication is similar to a key exchange in symmetric-key cryptography (e.g., one-time pad), an important distinction is that public-key cryptography techniques cannot be used to exchange this secret on a channel monitored by Willie without revealing the intention to communicate.
}

that we discuss next. We note that the fundamental results that follow apply not only to the classical spread-spectrum systems, but also to the modern covert communication proposals that rely on channel noise and equipment imperfections to hide communications (as is done, in, e.g., [5]).

\section{B. Square Root Law for Covert Communication over AWGN Channels}

Spread spectrum systems allow communication where it is prohibited because spreading the signal power over a large time-frequency space substantially reduces Willie's signal-tonoise ratio (SNR). This impairs his ability to discriminate between the noise and the information-carrying signal corrupted by noise. Here we determine just how small the power has to be for the communication to be fundamentally undetectable, and how much covert information can be transmitted reliably.

Consider an additive white Gaussian noise (AWGN) channel model where the signaling sequence is corrupted by the addition of a sequence of independent and identically distributed zero-mean Gaussian random variables with variance $\sigma^{2}$. This is the standard model for a free-space RF channel. Suppose that the channels from Alice to Bob and to Willie are subject to AWGN with respective variances $\sigma_{b}^{2}>0$ and $\sigma_{w}^{2}>04^{3}$ as illustrated in Figure 3a a). Let channel use denote the unit of communication resource-a fixed time period that is used to transmit a fixed-bandwidth signal — and let $n$ be the total number of channel uses available to Alice and Bob (e.g., $n=W_{s} T_{s}$ in Figure 2 b b ). Willie's ability to detect Alice's transmission depends on the amount of total power that she uses. Let's intuitively derive 4 Alice's power constraint assuming that Willie observes these $n$ channel uses. When Alice is not transmitting, Willie observes AWGN with total power $\sigma_{w}^{2} n$ over $n$ channel observations on average. By standard statistical arguments, with high probability, observations of the total power lie within $\pm c \sigma_{w}^{2} \sqrt{n}$ of this average, where $c$ is

\footnotetext{
${ }^{3}$ If the channel from Alice to Bob is noiseless $\left(\sigma_{b}^{2}=0\right)$ and the channel from Alice to Willie is noisy $\left(\sigma_{w}^{2}>0\right)$, then Alice can transmit an infinite amount of information to Bob; if the channel from Alice to Willie is noiseless $\left(\sigma_{w}^{2}=0\right)$, then covert communication is impossible.

${ }^{4}$ The formal proof is in 6 Section III].
} 


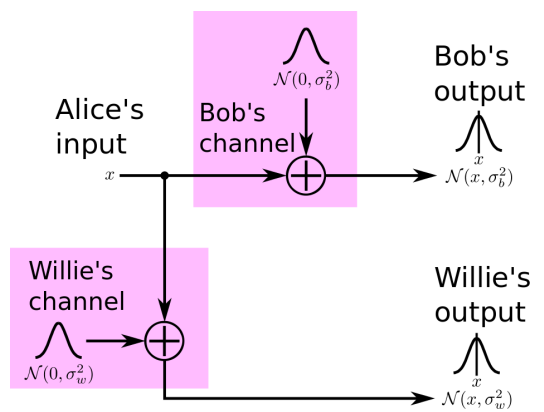

(a) AWGN channel

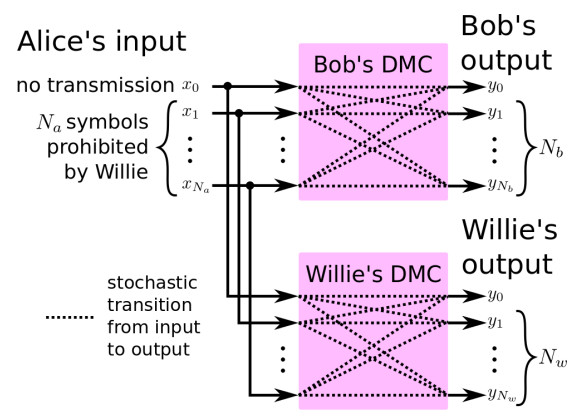

(b) DMC

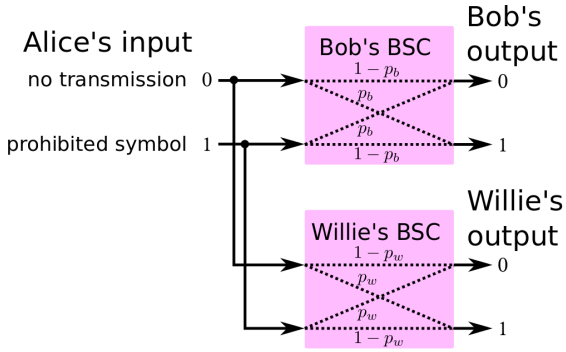

(c) BSC

Fig. 3: Channel models.

a constant. Since Willie observes Alice's signal power when she transmits in addition to the noise power, to prevent Willie from getting suspicious, the total power that Alice can emit over $n$ channel uses is limited to $\mathcal{O}\left(\sigma_{w}^{2} \sqrt{n}\right)$; otherwise her transmission will be detected (in fact, a standard radiometer suffices for Willie to detect her if she emits more power, provided $\sigma_{w}^{2}$ is knowr ${ }^{5}$. This allows her to reliably transmit $\mathcal{O}\left(\sigma_{w}^{2} \sqrt{n} / \sigma_{b}^{2}\right)$ covert bits to Bob in $n$ channel uses, but no more than that [6]. Note that, just like the steganographic square root law from Section II this yields a zero-rate channel (as $\lim _{n \rightarrow \infty} \frac{\mathcal{O}(\sqrt{n})}{n}=0$ bits/symbol). The similarity of this square root law for covert communications to the steganographic square root law is attributable to the mathematics of statistical hypothesis testing. The additional $\log n$ factor in the steganographic square root law comes from the fact that the steganographic "channel" to Bob is noiseless.

As in steganography and spread spectrum communication, prior to communicating, Alice and Bob may share a secret. For example, a scheme described in [6] and depicted in Figure 4 allows Alice and Bob to reliably transmit $\mathcal{O}\left(\sigma_{w}^{2} \sqrt{n} / \sigma_{b}^{2}\right)$ covert bits using binary amplitude modulation, any error-correction code (which can be known to Willie), and $\mathcal{O}(\sqrt{n} \log n)$ preshared secret bits. The secret contains a random subset $\mathcal{S}$ of $n$ available channel uses (effectively a frequency/timehopping pattern), and a random one-time pad of size $|\mathcal{S}|$. $\mathcal{S}$ is generated by flipping a biased random coin $n$ times with probability of heads $\mathcal{O}(1 / \sqrt{n})$ : the $i^{\text {th }}$ channel use is selected for transmission if the $i^{\text {th }}$ flip is heads; on average, $|\mathcal{S}|=\mathcal{O}(\sqrt{n})$. Knowledge of $\mathcal{S}$ allows Bob to discard the observations that are not in $\mathcal{S}$ and decode Alice's message; Willie observes mostly noise since he does not have $\mathcal{S}$. Rather than protecting the message content, the one-time pad prevents Willie's exploitation of the error correction code's structure to detect Alice.

While the size of the key is asymptotically larger than the size of the transmitted message, there are many realworld scenarios where this is an acceptable trade-off to being detected. Furthermore, the recent extension of [6] to digital covert communication that we describe next suggests that the pre-shared secret can be eliminated in some scenarios.

\footnotetext{
${ }^{5}$ See $[6$ Section IV] for the proof.
}

\section{Digital Covert Communication}

The discrete memoryless channel (DMC) model describing digital communication often sheds light on what is feasible in practical communication systems. DMC model assumes discrete input and output, which allows the DMC to be represented using a bipartite graph where the two sets of vertices correspond to input and output alphabets, and edges correspond to the stochastic transitions from input to output symbols. The memoryless nature of the DMC means that its output is statistically independent from any symbol other than the input at that time. We illustrate this model in Figure 3 b), which we augment by designating one of Alice's inputs as "no transmission"- a necessary default channel input permitted by Willie 6

We first consider the binary symmetric channel (BSC) illustrated in Figure 3 c), which restricts the DMC to binary input and output alphabet $\{0,1\}$, and the probability of a crossover from zero at the input to one at the output being equal to that of a crossover from one to zero. Denote by $p_{b}>0$ and $p_{w}>0$ the crossover probabilities on Bob's and Willie's BSCs, respectively. It has been shown that, while no more than $\mathcal{O}(\sqrt{n})$ covert bits can be reliably transmitted in $n$ BSC uses, if $p_{w}>p_{b}$, then the pre-shared secret is unnecessary [7].

Channel resolvability can be employed to generalize the square root law in [7] to DMCs. Channel resolvability is the minimum input entropy 7 needed to generate a channel output distribution that is "close" (by some measure of closeness between probability distribution 8 , to the channel output distribution for a given input; resolvability has been used to obtain new, stronger results for the information-theoretic secrecy capacity [8]. If the channels from Alice to both Willie and Bob are DMCs, and Willie's channel is worse than Bob's, then techniques in [7], [9] can be used to demonstrate the square root law without a pre-shared secret [10]. Furthermore, as

\footnotetext{
${ }^{6}$ For example, this could be the zero-signal in the AWGN channel scenario.

${ }^{7}$ Essentially, entropy measures "surprise" associated with a random variable, or its "uncertainty". For example, a binary random variable describing a flip of a fair coin with equal probabilities of heads and tails has higher entropy than the binary random variable describing a flip of a biased coin with probability of heads larger than tails. The output of the biased coin is more predictable, and less surprising, as one should observe more heads. Introductory texts on the information theory provide the in-depth discussion of entropy and other information-theoretic concepts.

${ }^{8}$ Examples of measures of closeness are variational distance and relative entropy.
} 


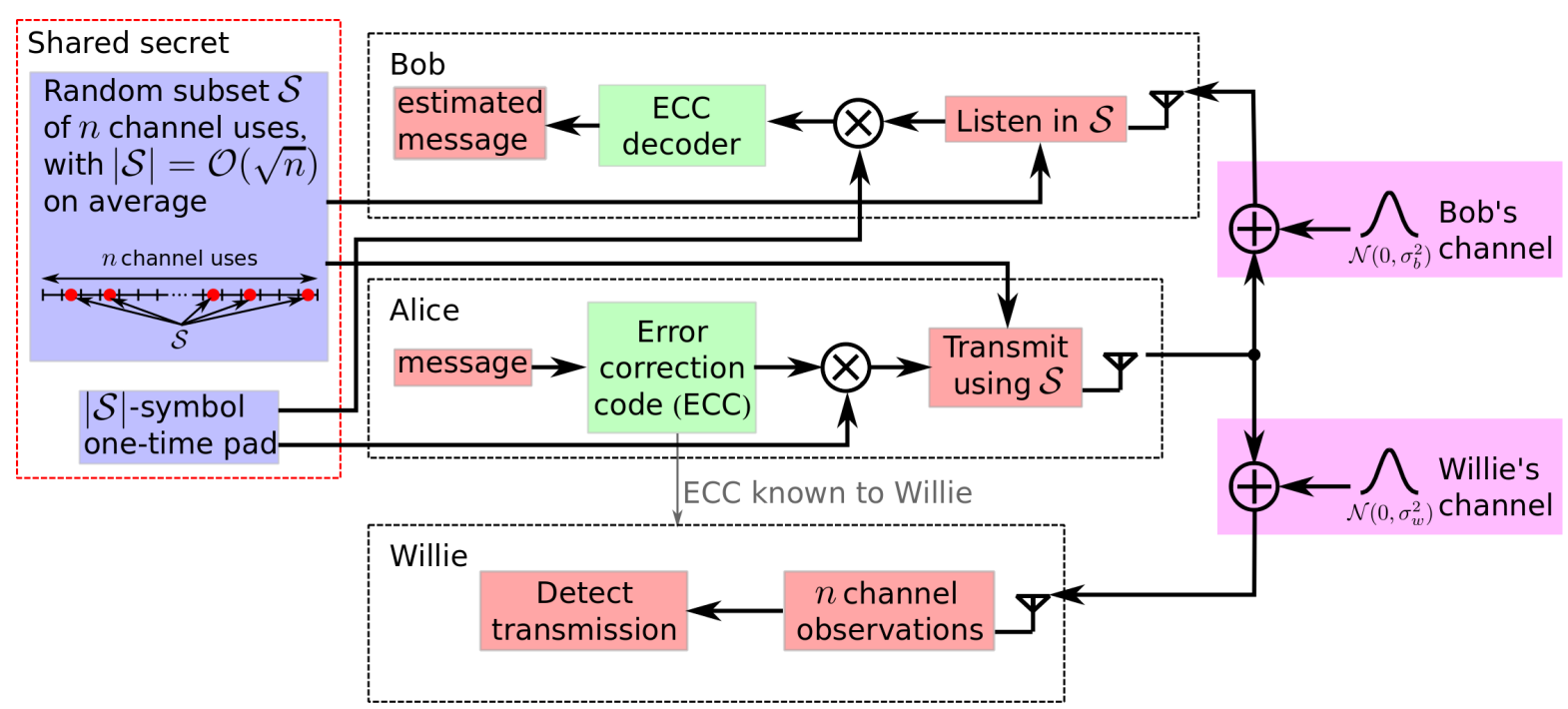

Fig. 4: Design of a covert communication system that allows Alice and Bob to use any error-correction codes (including those known to Willie) to reliably transmit $\mathcal{O}(\sqrt{n})$ covert bits using $\mathcal{O}(\sqrt{n} \log n)$ pre-shared secret bits.

long as the Alice-to-Willie channel is known to Alice, $\mathcal{O}(\sqrt{n})$ pre-shared secret bits are sufficient for covert communication when Willie's channel capacity is greater-than-or-equal to Bob's [10]. The results in [10] can be adapted to AWGN channels as well: a covert communication scheme exist: 9 that uses $\mathcal{O}(\sqrt{n})$ pre-shared secret bits, and, if the noise power at Willie's receiver is greater than that at Bob's receiver, then secret-less covert communication is achievable.

\section{Willie's Ignorance of Transmission Time Helps Alice}

When deriving the square root laws, we assume that Willie knows when the transmission takes place, if it does. However, in many practical scenarios Alice and Bob have a pre-arranged time for communication that is unknown to Willie (e.g., a certain time and day). The transmission might also be short relative to the total time during which it may take place (e.g., a few seconds out of the day). If Willie does not know when the message may be transmitted, he has to monitor a much longer time period than the time required for the transmission. It turns out that Willie's ignorance of Alice's transmission time allows her to transmit additional information to Bob. Surprisingly, under some mild conditions on the relationship between the total available transmission time and the transmission duration, Alice and Bob do not even have to pre-arrange the communication time. The technical details of this work are provided in [11].

\section{E. Positive-rate Covert Communication}

The covert communication channels described above are zero-rate, since the average number of bits that can be covertly transmitted per channel use tends to zero as the number of channel uses $n$ gets large. Here we discuss the possibility

\footnotetext{
${ }^{9}$ Conceptually the covert communication scheme that uses $\mathcal{O}(\sqrt{n})$ secret bits resembles the method that uses $\mathcal{O}(\sqrt{n} \log n)$ secret bits as described in Figure 4 and Section III-B however, its mathematical analysis is highly technical and is outside the scope of this article.
}

of positive-rate covert communication, i.e. reliable transmission of $\mathcal{O}(n)$ covert bits in $n$ channel uses. In general, the circumstances that allow Alice to covertly communicate with Bob at positive rates occur either when Willie allows Alice to transmit messages containing information (rather than zerosignal) or when he is ignorant of the probabilistic structure of the noise on his channel (note that the applicability of the steganographic results [2] here is limited since estimation of the probabilistic structure of the noise on Willie's channel is insufficient unless Alice can "replace" this noise rather than add to it). When Willie allows transmissions, the covert capacity is the same as the information-theoretic secrecy capacity (see [9] for treatment of the DMCs). Incompleteness of Willie's noise model can also allow positive-rate covert communication: in the noisy digital channel setting, Willie's ignorance of the channel model is a special case of the scenario in [9]; while in the AWGN channel setting, random noise power fluctuations have been shown to yield positive-rate covert communication [12]. The latter result holds even when the noise power can be bounded; a positive rate is achieved because Willie does not have a constant baseline of noise for comparison.

\section{F. Covert Broadcast}

Some of the results for the point-to-point covert communication in the presence of a single warden that are discussed in this section can easily be extended to scenarios with multiple independently-controlled receivers. For example, covert communication over an AWGN channel effectively imposes a power constraint on Alice. Since a pre-shared secret enables covert communication in this setting, if each receiver obtains it prior to communication, Alice can use standard techniques from network information theory to encode covert messages to multiple recipients. The extension to a multi-warden setting as well as other networked scenarios is the ongoing work discussed next. 


\section{CONCLUSION: TOWARDS Shadow NeTwORKS}

Our ultimate objective is to enable a wireless "shadow network", illustrated in Figure 11. comprised of transmitters, receivers, and friendly jammers that generate artificial noise, impairing wardens' ability to detect transmissions. While the relays are valuable and require protection, the jammers can be cheap, numerous, and disposable (i.e., the adversary can silence a particular jammer easily, but, because of their great numbers, silencing enough of them to produce a significant impact is infeasible). Thus, jammers have been shown to facilitate information-theoretically secrecy by confusing the eavesdropper even while being completely ignorant of the messages exchanged by legitimate communicating parties [13].

In covert networks jammer activities are independent from the relay transmission states: that is, wardens cannot detect transmissions by listening to the jammers. Thus, jammers have a parasitic effect on the wardens' SNRs and are a nuisance. It is important to characterize the scaling behavior of such a network, akin to the recent results for the secure (but not covert) multipath unicast communication in large wireless networks [14]. The first step towards this goal is extending the covert communication scenario of this article to point-to-point jammer-assisted covert communication in the presence of multiple wardens. Preliminary results [15] assume that jammers operate at a constant power, and the signal propagation model accounts only for path loss and AWGN. However, as [12] demonstrates, uncertainty in noise experienced by the warden is beneficial to Alice. Thus, variable jamming power and multipath fading should be incorporated into the jammer-assisted covert communication model, as it may enable covert communication at a positive rate. Completing the characterization of the point-to-point covert link in a multi-warden multi-jammer environment is an important step towards understanding the behavior of "shadow networks", and their eventual implementation.

\section{ACKNOWLEDGEMENT}

We are grateful to Matthieu Bloch, Gerhard Kramer, and Sid Jaggi for discussions and comments on a draft of this article.

\section{REFERENCES}

[1] J. Fridrich, Steganography in Digital Media: Principles, Algorithms, and Applications, 1st ed. New York: Cambridge University Press, 2009.

[2] S. Craver and J. Yu, "Subset selection circumvents the square root law," vol. 7541, 2010, pp. 754 103-1-754 103-6.

[3] M. K. Simon, J. K. Omura, R. A. Scholtz, and B. K. Levitt, Spread Spectrum Communications Handbook. McGraw-Hill, 1994.

[4] A. O. Hero, "Secure space-time communication," IEEE Trans. Inf. Theory, vol. 49, no. 12, pp. 3235-3249, Dec. 2003.

[5] A. Dutta, D. Saha, D. Grunwald, and D. Sicker, "Secret agent radio: Covert communication through dirty constellations," in Proc. 14th Int. Conf. Inform. Hiding, ser. IH'12, Berkeley, CA, 2013, pp. 160-175.

[6] B. A. Bash, D. Goeckel, and D. Towsley, "Limits of reliable communication with low probability of detection on AWGN channels," IEEE J. Selected Areas Commun., vol. 31, no. 9, pp. 1921-1930, 2013, Originally presented at ISIT 2012, Cambridge MA.

[7] P. H. Che, M. Bakshi, and S. Jaggi, "Reliable deniable communication: Hiding messages in noise," arXiv:1304.6693 2013.

[8] M. Bloch and J. Laneman, "Strong secrecy from channel resolvability," IEEE Trans. Inf. Theory, vol. 59, no. 12, pp. 8077-8098, Dec 2013.
[9] J. Hou and G. Kramer, "Effective secrecy: Reliability, confusion and stealth," in Proc. of IEEE Int. Symp. Inf. Theory (ISIT), Honolulu, HI, Jul. 2014, arXiv:1311.1411

[10] M. Bloch, "Covert communication over noisy memoryless channels: A resolvability perspective," arXiv:1503.08778 2015.

[11] B. A. Bash, D. Goeckel, and D. Towsley, "LPD Communication when the Warden Does Not Know When," in Proc. of IEEE Int. Symp. Inf. Theory (ISIT), Honolulu, HI, Jul. 2014, arXiv:1403.1013

[12] S. Lee, R. Baxley, M. Weitnauer, and B. Walkenhorst, "Achieving undetectable communication,” IEEE J. Sel. Topics Signal Proc., 2015, to appear.

[13] L. Lai and H. El Gamal, "The relay-eavesdropper channel: Cooperation for secrecy," IEEE Trans. Inf. Theory, vol. 54, no. 9, pp. 4005-4019, Sept 2008.

[14] C. Capar, D. Goeckel, B. Liu, and D. Towsley, "Secret communication in large wireless networks without eavesdropper location information," in 2012 Proceedings of IEEE INFOCOM, March 2012, pp. 1152-1160.

[15] R. Soltani, B. A. Bash, D. Goeckel, S. Guha, and D. Towsley, "Covert single-hop communication in a wireless network with distributed artificial noise generation," in Proc. of Conf. on Commun., Control, Comp. (Allerton), Monticello, IL, 2014.

Boulat A. Bash holds BA (2001) in Economics from Dartmouth College, and MS (2008) and $\mathrm{PhD}$ (2015) in Computer Science from the University of Massachusetts, Amherst. He is currently a Postdoctoral Fellow in the Quantum Information Processing (QuIP) group at Raytheon BBN Technologies. His research interests include security, privacy, communications, signal processing, and information theory.

Dennis Goeckel holds BS (1992) from Purdue University, and MS (1993) and $\mathrm{PhD}$ (1996) from the University of Michigan. He is a Professor in the Electrical and Computer Engineering Department at the University of Massachusetts, Amherst. His research interests include physical layer communications and wireless network theory. He received the NSF CAREER Award (1999) and is an IEEE Fellow. He was a Lilly Teaching Fellow (2000-2001), and received the University of Massachusetts Distinguished Teaching Award (2007).

Saikat Guha holds BTech in Electrical Engineering (2002) from the Indian Institute of Technology, Kanpur, and SM (2004) and PhD (2008) in Electrical Engineering and Computer Science from the Massachusetts Institute of Technology. He is a Senior Scientist in the Quantum Information Processing (QuIP) group at Raytheon BBN Technologies. His research interests span quantum-optical communication and sensing, optical quantum computing, and network information theory. He received a NASA Tech Brief Award in 2010.

Don Towsley holds BA (1971) in Physics and PhD (1975) in Computer Science from University of Texas. He is a Distinguished Professor at the University of Massachusetts in the School of Computer Science. His research interests include networks and network science. He received several achievement awards including the 2007 IEEE Koji Kobayashi Award and numerous paper awards. He is a Fellow of both the ACM and IEEE. 